\title{
Utilization of Cloud Computing in Education and Research to the Attainment of Millennium Development Goals and Vision 2030 in Kenya
}

\author{
Duncan Waga ${ }^{1, *}$, Esther Makori $^{2},{\text { Kefa } \text { Rabah }^{3}}^{3}$ \\ ${ }^{1}$ Jaramogi Oginga Odinga University of Science and Technology, Kenya \\ ${ }^{2}$ Kisii University, Kenya \\ ${ }^{3}$ Kabarak University, Kenya \\ *Corresponding Author: wagadun@gmail.com
}

Copyright (C) 2014 Horizon Research Publishing All rights reserved.

\begin{abstract}
Kenya Educational and Research fraternity has highly qualified human resource capacity with globally gained experiences. However each research entity works in disparity due to the absence of a common digital platform while educational units don't even have the basic infrastructure. For sustainability of Education and research progression, collaboration amongst students, Teachers, Lecturers and International researchers on a common central repository of digital content is a requirement. The absence of a centralized digital content repository containing e-learning resources, research applications and tools with a collaborative on-line modern digital library accessible with a controlled right based accessibility has denied students in remote areas access to education, researchers from collaborating with their peers and industry from innovation benefits. This study proposes a framework of cloud computing (CC) owned and maintained by the government. The developed repository may need to be accessed by any terminal that can be easily solar powered and may use basic modems. Cloud provider preference guided by digital content development capacity is discussed. Students will benefit immensely on the e-learning component which will address the dwindling instructors-Student ratios while universities and research institutes through collaborative ventures will participate alongside their international colleagues with immediate innovation benefiting our local industries. Contribution to Kenya's vision 2030 by Millennium development goals (MDGs) attainment like universal primary education, environmental sustainability and combating diseases through research is discussed. Challenges of instructor shortage, classroom space and geographical disparity are addressed.
\end{abstract}

Keywords Common Digital Platform, Cloud Computing, Millennium Development Goals, Vision 2030, E-Learning, Geographical Disparity

\section{Introduction}

Education and Research are key sectors targeted by Kenya's Vision 2030 blueprint which closely relates to the Millennium Development Goals (MDGs). Science, Technology and Innovation is a key foundation to Vision 2030 due to its capacity to enhance the attainment of the other pillars. The absence of a common digital platform for collaboration hampers this capacity. CC implementations in these sectors with a package of e-learning, research portal connected to online libraries will not only fast track attainment but endear the country towards a research and innovation based economy.

In Kenya, Researchers work in isolation and often less innovative due to the high cost of their trade while educationist suffer from classroom shortage, few qualified teachers and absence of proper teaching aids. There is a tremendous gap of absence of a digital platform that unites these two noble sectors. As a result, each work in disparity. The immediate effect of this is the country's high cost of living caused by the local industry independence on technology importation due to the absence of innovation. CC, a utility way of service consumption of centralized computing resources loaded with an e-learning component with Research portal promises to deliver such a missing platform hence the significance of this study.

This study will demonstrate how cloud computing will provide this platform and thereafter enhance the attainment of the millennium development goals which culminates to the vision 2030 Kenya has set to achieve. Pillars of the MDGs like eradication of extreme poverty, primary education for all and combating HIV Aids and Malaria achievement is enhanced by this framework due to the fact that researchers will collaboratively interact on one common platform as cost of education goes down owing to the shared nature of the pay as you use model of cloud computing.

Industry practitioners could also collaborate with universities on the same platform resulting into production of 
labor focused professionals. This is reiterated further by Nabil Sultan (2010) in his cloud computing for education journal where he found out that cloud computing is the anecdote that the cash strapped education establishments has been waiting for with immense benefits.

Wen-Hsi Lydia Hsu (2012) in his conceptual framework article of cloud computing governance model-An education perspective concurs that education establishments can more effectively govern their cloud computing related risks and integrates education with learning in one model with enhanced corporate performance.

\section{Cloud Definition}

Clouds are medium sized computing models that enable ubiquitous, convenient, on-demand network access to a shared pool of configurable computing resources (e.g., networks, servers, storage, applications, and services) that can be rapidly provisioned and released with minimal Management effort or service provider interaction, Peter Mell et al(2009) . Cloud computing generally comes in three different approaches i.e. private, public and community clouds of which various environments can be implement i.e. Software as a service (SAAS), Platform as a service(PAAS) and Infrastructure as a service(IAAS).

This approach is intended to engage specific cloud computing provider whose capacity is rated depending on its ability to develop specific digital content, and its position in the global CC provider market. They should be able to provide SaaS and PaaS cloud types through which e-learning modules are deployed. Stakeholders like students, teachers and researchers will use solar powered terminals to port into this private cloud through the internet of which they could use basic modems. Other content will be research specific Apps and tools and education related content approved by the government. Remote schools will benefit immensely since they will be able to access resources which would otherwise been out of reach and so is researchers. The purpose of this study is to demonstrate how countries like Kenya can employ cloud computing in its education and research sectors and quickly attain the MDGs and vision 2030.

\section{Literature Review}

Contrary to popular opinion that cloud computing is a new concept, companies known as Application Service Providers (ASP) since 1990s have been providing business services via the internet. However due to the cost of bandwidth, the utility nature did not take off (Carr, 2009). This was followed by XML Web services that represented a model of software delivery based on the notion that pieces of software applications can be developed and then published to a registry where they can be used by other client applications over different transport protocols irrespective of the language used to develop those applications or the platforms on which they are implemented. The ability to automate the process of discovery, binding, and invocation of Web services on the Internet without human intervention (Manes, 2004; Nakhimovsky\& Myers, 2004) received a great deal of hype.

One technology analyst and author (David Chappell) in 2003 even doubted if there was a business case for Web services (Chappell, 2003). However, Web services are nowhere near achieving the full potential that was hoped for. Nevertheless, the technology is being implemented successfully (and commercially) by many of the big players such as eBay, Amazon and Google (Iskold, 2006). Furthermore, the technology has also created the foundation for a new Enterprise Application Integration (EAI) paradigm known as Service-Oriented Architecture (SOA). The extensible XML-based nature of SOAP has enabled many organizations to expose some of their legacy and disparate systems as Web services in order to achieve total integration of their systems (Clark, 2007; Flinders, 2007; Mohamed, 2007).Most importantly, SOAP-based Web services are now being used in the delivery of some aspects of a new computing paradigm (namely cloud computing) which not only promises to deliver software remotely but also other computing-related functionality thanks also to other relatively new technologies such as Virtualization and grid computing.

This paper comes at a time the Kenya government has set aside huge resources from budget (tax payers money) to a project whose chances of success are limited. The economically struggling government is about to roll out a non viable and expensive laptop project to schools for class one pupils. Analysts say this is more of an ego project since the leaders are basically trying to outdo their opponents by showing that they can meet whatever promises they had made to the electorate. "We all hope that laptop provision to pupils will miraculously seal the digital divide", said one proponent. The IS research fraternity has been fundamentally been left out in this venture although their main role should have been to give direction to technical matters such as this and that's why the government crafted a Ministry of Information and Communication Technology to spearhead government IS technical ventures.

Nabil Sultan, in his work published on the International Journal of Information Management, Cloud computing for education dwelt at length on cloud computing in the education sector a case of schools in Ethiopia and covered aspects of cost savings being realized with this phenomenon. Technology flow in his project evaluates previous Information and Communication Technology policy and practice relating to primary schools in Ireland with a view to suggesting a better way forward in light of advances in ICT, such as the availability of fast broadband services, including fiber broadband, browser-based applications and the advent of cloud computing. Junjie Peng in his work on Comparison of Several Cloud (2009) Computing Platforms considered various platforms of cloud and demonstrated how suitable clouds are to organizations. Since funds have already been 
set aside for a project which has all the indications of failure Kenya can exploit this opportunity to come up with an integrated cloud platform not only useful to the class one project but also to more valuable research in high schools, universities and institutes. In this region Kenya has been cited to be one of the countries whose ICT development ranks high especially with their electronic funds transfer, Mpesa. Kenya as a country is at an economic cross road of implementing the new constitution and any savings would go a long way. This framework would eventually be integrated with the e-government project with great benefits. One great limitation is the politics involved which may hinder implementation. Other challenges like efficient power availability and building Teacher capacity and digital content are also considered in the paper.

The main concern in this research is the fact that a government is about to waste time and money on a non viable project despite the availability of technical and human resources. The opportunity can be exploited to serve a wider population with tremendous benefits. In this paper we will investigate the viability, cost effectiveness and suitability of clouds in education as opposed to stand alone infrastructures. A framework which is inclusive of all learning institutions of the country with ease of connectivity to a platform of e-learning, computing applications and tools and research portal complete with an online library would be a more viable venture. If implemented huge cost savings will be realized which could be channeled to its inclusion of the universities and research institute component.

Krautheim in his work on cloud security discusses a more secure framework where the client and the provider share the securing aspects due to the involvement of many players. Taylor in Computer Law \& Security Review elaborates his research on digital forensics which favor clouds since much dispersed digital resources makes a nightmare on investigations especially when various jurisdictions are included. Due to the newness of cloud technology, very little research has been ongoing and most are just scratching the surface as the technology permeates. A few works like ShaikSaidhbi's in Ethiopia which looked at CC in education and research showed that Cloud Computing is the better ICT utilization mechanism for Education institutions teaching-learning and service delivery requirements, for it enables wise and strategic use of technology which significantly reduces cost.

This paper's originality stems from the fact that it significantly touches on the contribution of the MDGs. Clouds are also known to be more secure and trusted due to the service level contracts entered to by the stakeholders hence the higher uptake rates by enterprises. This investigation will directly benefit the educational fraternity including research institutes and universities. Provision of unreachable technology will be a main benefit besides security and data confidentiality. It has been a common joke that 7 year old pupils will carry heavy laptops to and fro and will merely play games since their teachers don't have the capacity to deliver on the content which is yet to be agreed on and developed. This framework intends to engage the providers whose products have been used in "a content and capacity development program" to ensure the implementation is a success. The main limitation will be the politics involved since the leaders would want to see their initial political promise to the electorate being implemented without dilution.

\section{Advantages of the Cloud}

Security - Major cloud providers like the Drop box, Amazon Cloud Service, and GoGrid among others have each suffered from security breaches that have negatively impacted their customers' reputations and bottom lines.

Revenue Generation - There are many opportunities to generate income through cloud computing.

Virtualization - The ability to virtualize services maximizes hardware usage, ultimately reducing costs and complexity.

Researchers would collaborate more effectively by having in place a common portal connected to on line libraries. Resultant Innovation would benefit industry while local without capacity to acquire systems would enjoy them freely without any upfront investment. Data and resources are the most treasonable items to an organization and hosting them to $3^{\text {rd }}$ parties would pose a challenge. It is therefore an internal responsibility to manage this risk. Therefore until more is done on the public cloud infrastructures, the private cloud still remains the most secure.

Well developed content can only be coordinated by government and provision of the same to the stakeholders is the way to go. As opposed to the standalone laptops which would suffer the brunt of being carried daily in terms of wear and tear and loss. This framework has secure infrastructure and only can be accessed from cheap terminals. It is intended that Green IT be embraced by the use of solar powered VDIs and the content hosted in green datacenters. This framework hopes to be integrated to the e-government system which would impressively put the country forward in terms of research and service delivery.

\section{Methodology}

Literature search was used to study related works and have a comparison based on different studies and environments. Cloud computing providers were selected according to their capacity to handle large government projects and their global vendor position. Cloud environments selected must have IaaS, SaaS and PaaS capacity. On these, E-learning component is integrated, Research Apps and Tools besides other education based digital content approved by the government. This study significantly diverts the resources from the promised laptops to a framework where they are used to setup a VDI to access the government owned cloud. Virtual desktop infrastructure (VDI) is the practice of hosting a desktop operating system within a virtual machine 
(VM) running on a centralized server. VDI is a variation on the client/server computing model, sometimes referred to as server-based computing. The term was coined by VMware Inc. In the past couple of years, some large organizations have turned to VDI as an alternative to the server-based computing model used by Citrix and Microsoft Terminal Services. (see Figure 1)

\section{E-Learning}

E-Learning is electronic learning, in which the learner uses a computer to learn a task, skill, or process. It is also referred to as computer-based training, web-based training, and online learning(Digital Harbor). E-Learning can be even more effective than traditional, classroom learning according to the report prepared for the U.S. Department of Education in August 2009. Another report by the ASTD organization recommended that to survive these tough economic times, companies may want to replace some of their instructor-led training with eLearning. As a component it is plugged into the framework to take care of the educational module. It comes in four categories i.e. lower primary, upper primary, secondary and tertiary in which respective content for these levels are found. Content is developed by the $\mathrm{CC}$ providers due to their financial muscle and experience. With e-learning education can be delivered and received anywhere and anytime.

\section{Virtual Desktops}

1. Access machines from anywhere via an RDP-enabled device. Use a laptop, desktop, tablet or smart phone from the office, at home or on the road.

2. Run applications and systems that a device would not ordinarily be able to run (i.e. run Windows on an iPad(R).

3. Allow employees to use whatever device they want with a Bring Your Own Device (BYOD) model without worrying that their device be precisely configured to work with the company's systems.

4. Purchase thin clients. Thin clients cost a fraction of fully-provisioned machines and have a lifespan twice as long. They are also cheaper and easier to maintain.

5. Make system changes and upgrades quickly and easily from the Cloud Management Center, rather than manually making changes on each individual machine.

\section{Conceptual Framework}

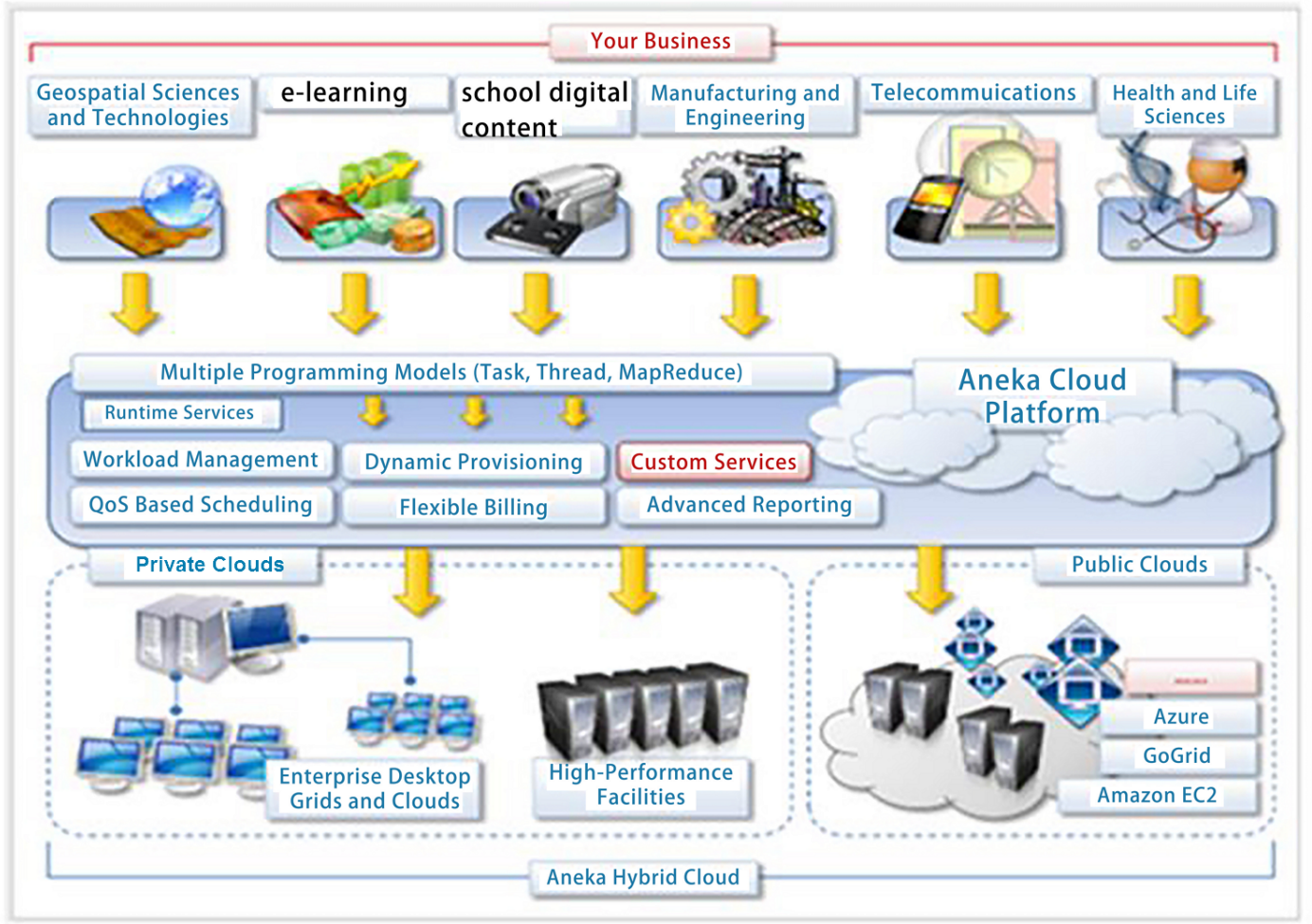

Source: Manjrasoft pty ltd

Figure 1. Model integrating e-learning and digital content modules 


\section{Green IT}

High concentrations of conventional laptops would have pumped out too much heat for the natural air cooling system to deal with. A green IT solution cloud clients, consumes a fraction of the electricity compared to a standard PC and hardly generates any heat. In fact each Dell Wyse cloud client computer uses approximately $10 \%$ or less of the power required by a laptop and generates less heat than the person using it. The cloud clients must be easy to manage remotely and deliver a user experience equivalent, if not better than using a laptop. VDI solution with cloud clients fits this bill perfectly. There also is added security because the cloud clients are useless unless connected to the central servers, which is important in the open learning spaces. Citrix software is used to publish desktop applications to each cloud client terminal. All of the standard Microsoft applications plus some specialist statistical software are made available via a user's personal login detail, which also provides access to personal data. The same software supports desktop anywhere, a service that allows staff and students to access their personal applications and data offsite.

The design and cost efficiencies of the Dell Wyse solution will enable the school project to flood numerous cloud clients and create new areas for accessible learning. The public areas rely on environmentally friendly air cooling rather than traditional air conditioning. The much lower heat output of the cloud clients therefore was an essential part of this work which had to take into account the thermal discharge of both people and laptops. The datacenters which host the digital content is professionally managed and observes principles of green IT.

\section{Moving to the Cloud}

The proposed framework will provide a distributed computing environment for entire Schools, colleges and institutions in Kenya as well as extended environments, thereby creating further economies of scale by eliminating redundant resources and centralizing and simplifying maintenance for over-burdened IT staff. Schools and campuses can be connected using secure WAN links, and centralized servers and software resources can be accessed using virtual desktops located anywhere. With the proliferation of cloud-based applications, students can take advantage of collaborative solutions such as Google Apps for Education, invite their classmates to review their work, and complete their work in the lab, the classroom, or at home. For support staff, a cloud-based system can substantially reduce their load, leveraging efficiencies across the nationwide or statewide school network. The problem of overcrowded classrooms or distance learning challenges can be surmounted through virtual classrooms, with students attending class in their own homes on their own computers, with the teacher being present hundreds of miles away. Most schools will not have sufficient hardware or software to provide students a complete learning experience. This problem is especially pronounced in the technical and science fields.

However, cloud-based approaches using virtual desktop infrastructure can pave the way by enabling access to resources that would be impractical to provide in a PC-based environment. Professors would deliver their course content while travelling etc. Some countries are already moving in this direction. In early 2011, the Higher Education Funding Council for England announced a plan to allocate $£ 12.5$ million to a new program that will fund shared services in cloud computing at colleges and universities across the country.

\section{Findings}

There is no arguing that "cloud computing" is gaining a great deal of momentum. Worldwide, cloud services revenue is forecast to reach $\$ 68.3$ billion in 2010 , a $16.6 \%$ increase from 2009 revenue of $\$ 58.6$ billion, according to analyst firm Gartner Inc. So what does this mean to the accounting profession? What are the benefits and risks? Who are the vendors in the proverbial sky, and how do you know you can trust them with your data - or your clients' data, for that matter cloud computing enables the sharing of it resources, making the total cost of a service variable with use (pay as you go). This enables it cost to be funded as an expense rather than as an investment. Jiaqiduan et al in proceedings of the 2012-13 courses on advanced resource planning summarized the benefits and drawbacks of cloud-based versus traditional systems as detailed in the table 1 below: 
Table 1. Comparison of features of cloud-based, hosted and on-premise ERP

\begin{tabular}{|c|c|c|c|}
\hline Relevant Distinctive Features & $\begin{array}{l}\text { Cloud- } \\
\text { based ERP }\end{array}$ & $\begin{array}{l}\text { Hosted } \\
\text { ERP }\end{array}$ & $\begin{array}{l}\text { On-premise } \\
\text { ERP }\end{array}$ \\
\hline \multicolumn{4}{|l|}{ Direct Costs } \\
\hline $\begin{array}{l}\text { Lower upfront costs (hardware, user licenses, implementation,ex- } \\
\text { cluding training and customization) }\end{array}$ & $x x x$ & $x$ & \\
\hline $\begin{array}{l}\text { Lower operating costs and efforts (energy, maintenance, configur- } \\
\text { ing, upgrades,IT staff costs) }\end{array}$ & $x x x$ & $x$ & \\
\hline No non-depreciable subscription fees & & $x$ & $x x$ \\
\hline \multicolumn{4}{|l|}{ Impact on Competitive Advantages and Organization } \\
\hline $\begin{array}{l}\text { Scalability (highly elastic infrastructure capacity). faster time to } \\
\text { market }\end{array}$ & xxx & & \\
\hline Rapid implementation, casier to switch among IT providers & $\mathrm{xxx}$ & $x$ & \\
\hline Enables enhanced focus on core competencies & $x x$ & & \\
\hline Higher level of independency from the ERP provider & & & $x x$ \\
\hline Minimized loss of IT skills and IT staff resistance to changes & & & $x$ \\
\hline \multicolumn{4}{|l|}{ Functionality \& Usability } \\
\hline $\begin{array}{l}\text { Functionally rich to satisfy the back-office needs of organizations in } \\
\text { all types of industries }\end{array}$ & & $x x$ & $x x$ \\
\hline Rapid acquisition of bug fixes and new functionality & $\mathrm{xxx}$ & & \\
\hline Improved accessibility, mobility, and usability & $\mathrm{xxx}$ & $x$ & \\
\hline \multicolumn{4}{|l|}{ Integration \& Customization \& Performance Capabilities } \\
\hline Enables extensive customization and complex integration & & $x x$ & $\mathrm{xx}$ \\
\hline Easier integration with other cloud services & $x x$ & $x$ & \\
\hline Improved system disaster recovery and availability & $x$ & $x$ & \\
\hline Low dependency on deficiency of network reliability and speed & & & $x x x$ \\
\hline Ease of retaining on-premise legacy systems & & & $x x x$ \\
\hline Easier integration with on-premise systems that require low latency & & & $\mathrm{xx}$ \\
\hline Allows hybrid deployment strategy to incorporate 3 features above & & $\mathrm{xx}$ & \\
\hline \multicolumn{4}{|l|}{ Security \& Standards } \\
\hline Enables high level of security and confidentiality & $x$ & $x x$ & $\mathrm{xxx}$ \\
\hline Clear data damage liability and SLA conditions & & & $x$ \\
\hline Easier compliance to data \& environmental regulations & & $x$ & $\mathrm{xx}$ \\
\hline
\end{tabular}

Legend

$\mathrm{X}$ referred to (dedued from) only in one source, or pertinent only partially, or has noticeably contradictive references $\mathrm{XX}$ referred to (deduced from) in 2 to 5 sources

\section{MDGs and Vision 2030}

Vision 2030 is Kenya's development programme covering the period 2008 to 2030. It was launched on 10 June 2008 by President MwaiKibaki with an objective of helping transform Kenya into a newly industrializing, middle-income country providing a high quality of life to all its citizens by 2030 in a clean and secure environment. Developed through "an all-inclusive and participatory stakeholder consultative process, involving Kenyans from all parts of the country," the Vision is based around three "pillars": Economic, Social, and Political. The Vision's adoption comes after the country's GDP growth, from $0.6 \%$ in 2002 to $6.1 \%$ in 2006, under Kibaki's Economic Recovery Strategy for Wealth and Employment Creation (ERS). The Kenya Vision 2030 is to be implemented in successive five-year medium-term plans, with the first such plan covering the period 2008-2012 (with the next covering the period 2012-2017, and so until 2030). Under the Vision, Kenya expects to meet its Millennium Development Goals (MDGs) by the deadline in 2015, with some of them already met as posted in the Kenya vision 2030 website.

The Millennium Development Goals (MDGs) are eight international development goals that were officially established following the Millennium Summit of the United Nations in 2000, following the adoption of the United Nations Millennium Declaration. All 189 United Nations member states and at least 23 international organizations have agreed to achieve these goals by the year 2015 .

The goals are:

1. Eradicating extreme poverty and hunger,

2. Achieving universal primary education,

3. Promoting gender equality and empowering women,

4. Reducing child mortality rates,

5. Improving maternal health,

6. Combating HIV/AIDS, malaria, and other diseases, 
7. Ensuring environmental sustainability, and

8. Developing a global partnership for development.

9. Halve by 2015 the proportion of people without sustainable access to safe drinking water

Summarily, this study will have a major influence on the attainment of the MDGs above and consequently the actualization of the economic and social pillars of the vision 2030.The collaborative research capacity enhancement will enhance the attainment of the MDGs i.e. universal primary education, HIV Aids and malaria research and extreme poverty alleviation. Student in remote sites will easily access the content through simply solar powered terminals and enjoy content which would otherwise be beyond reach.

\section{Discussion}

This work relates closely to Wen - Hsi's conceptual framework where he elaborated on how a cloud computing framework in educational establishments can go a long way in ensuring corporate infrastructure is well set and secured to serve effectively. ShaikSaidhbi in his cloud computing work on educational institutions in Ethiopia found out the same advantages this paper found out. "Over the past few years, we might have seen VDI adoption only make sense for $5 \%$ to $10 \%$ of the enterprise desktops out there. Now that VDI can support 1-to-1 persistent disk images and graphically-intense applications, VDI becomes a valid option for 40 or $50 \%$ of enterprise desktops" argues Brian Madden, a Desktop virtualization expert and blogger.

Hence we may conclusively state that $\mathrm{CC}$ frameworks improve education standards and research and has a lot in store that can be exploited by governments despite the fact that there are network problems in Kenya although more corporates are beginning to invest in that area.

\section{Conclusion}

Cloud computing has been proved to be secure, cost effective and sustainable although further work is required to perfect this model. When implemented, significant tax payers money will be saved from buying the individual numerous laptops, save the environment through green IT besides leverage Technological Innovation. Cloud computing is the buzz word at the moment and if integrated with the educational sector including universities minus the politics, then the countries wanting research capacity will be enhanced. This is a rich area which should be able to attract donor funding for its further development and implementation especially when it is driving the country to industrialization and wealth creation besides quality living.

\section{REFERENCES}

[1] Norman P. Jouppi, "The Nonuniform Distribution of Instruction-Level and Machine Parallelism and Its Effect on Performance," IEEE Transactions on Computers, Vol. 38, No. 12, pp. 1645-1658, December 1989.

[2] M. Armbrust, et al., "A View of Cloud Computing," Communication of ACM, vol. 53, pp. 50-58, 2010. A. Lenk, et al., "What is Inside the Cloud? An Architectural Map of the Cloud Landscape," presented at the Workshop on Software Engineering Challenges of Cloud Computing, Collocated with ICSE 2009 Vancouver, Canada, 2009.

[3] L. Vaquero, et al., "A break in the clouds: towards a cloud definition," IGCOMM Computer Communications Review, vol. 39, pp. 50-55, 2009.

[4] P. Mell and T. Grance, "The NIST Definition of Cloud Computing," National Institute of Standards and Technology, USA2009

[5] Cloud computing for education: A new dawn? Nabil Sultan Faculty of Business and Computer Science, Liverpool Hope University, Hope Park, Liverpool, L16 9JD, UK Shane O'Doherty, of Dublin Institute of Technology

[6] Comparison of Several Cloud Computing PlatformsPrivate Virtual Infrastructure for Cloud Computing F. John Krautheim

[7] University of Maryland, Baltimore County, 1000 Hilltop Circle, Baltimore, MD 21250

[8] Digital evidence in cloud computing systems M. Taylor, J. Haggertyb, D. Gresty, R. Hegarty School of Computing and Mathematical Sciences, Liverpool John Moores University, UK.

[9] Improving Student Access to Technology in the Classroom -in a Declining Budget Environment, The Role of Cloud Computing and Virtualization in Education A white paper by Dell Wyse.

[10] Cloud computing for education: A new dawn? Nabil Sultan,Faculty of Business and Computer Science, Liverpool Hope University, Hope Park, Liverpool, L16 9JD, UK

[11] The NIST Definition of Cloud Computing,PeterMell

[12] Cloud Computing: What Accountants Need to Know : A journal of accountancy by Alexandra defeliceoctober 2010

[13] Shedding light on cloud computing A Primer By Gregor Petri

[14] Benefits and drawbacks of cloud-based versus traditional erpsystemsJiaqiDuanProceedings of the 2012-13course on Advanced Resource Planning W.J.H. van Groenendaal (ed.)

[15] First five steps to building a private cloud environment, Bob PlankersW. Anderson, F. J. Sparacio, and R. M. Tomasulo, "The IBM System/360 Model 91: Machine Philosophy and Instruction-Handling," IBM Journal of Research and Development, Vol. 11, No. 1, pp. 8-24, January 1967.Proceedings. Supercomputing '88 (IEEE Cat.No.88CH2617-9), November, 1988.

[16] Conceptual framework of cloud computing governance model -an education perspective by Wen-Hsi Lydia Hsu

[17] http://www.vision2030.go.ke/

[18] Cloudonomics the business value of cloud computing by Joe Weinman

[19] Solid Justifications for Cloud Computing -- and 10 Situations Where It Doesn't Work

[20] Conceptual framework of bio-cloud Computing prof. Swati vitkar, 2 prof. Vishwanath kale Cloud Computing for Higher Education: A RoadmapMasud et al 